\title{
Preface to the special issue of INCAM 2019 Conference on fluid mechanics
}

\author{
Arvind Pattamatta $^{1}$ - B. S. V. Prasad Patnaik ${ }^{1}$
}

Published online: 30 April 2020

(C) Indian Institute of Technology Madras 2020

Since its inception in 2016, the Indian Society of Applied Mechanics (ISAM) has been pivotal in organizing its flagship biennial event, the Indian Conference on Applied Mechanics (INCAM). It has been a unique platform in bringing together experts from academia and industry for the dissemination of ideas and sharing of knowledge broadly in the field of applied mechanics.

The 4th Indian Conference on Applied Mechanics (INCAM 2019) was jointly organized by Departments of Civil and Mechanical Engineering at the Indian Institute of Science Bangalore during July 3-5, 2019. Out of the total 146 contributed papers, 12 were related to fluid mechanics. These were carefully screened, and the selected ones were taken up for further review. Finally, six papers focusing on various topics of current interest in Fluid Mechanics were chosen after peer review for publication in this special issue of 'International Journal of Advances in Engineering Sciences and Applied Mathematics.' The topics covered under this special issue range from computational fluid mechanics of turbulent flows to Bio-Fluid mechanics, thus highlighting the recent advances in fluid mechanics research.

The guest editors appreciate the authors for their contributions and also thank all the reviewers for their prompt and critical comments. Special thanks are due to the Springer publishing team and the Editor-in-Chief, Prof. Harishankar Ramachandran for agreeing to bring out this special issue.

Guest Editors

Arvind Pattamatta

BSV Prasad Patnaik
Arvind Pattamatta

arvindp@iitm.ac.in

1 Indian Institute of Technology Madras, Chennai 600036, India 